\title{
Le défi de la sylviculture en faveur des produits forestiers non ligneux sous les tropiques : de la cueillette à l'agriculture?
}

\section{Paul Vantomme}

Sophia GAZZA

FAO, Département des forêts Division de l'économie, des politiques et des produits forestiers Via delle Terme di Caracalla 00100 Rome Italie

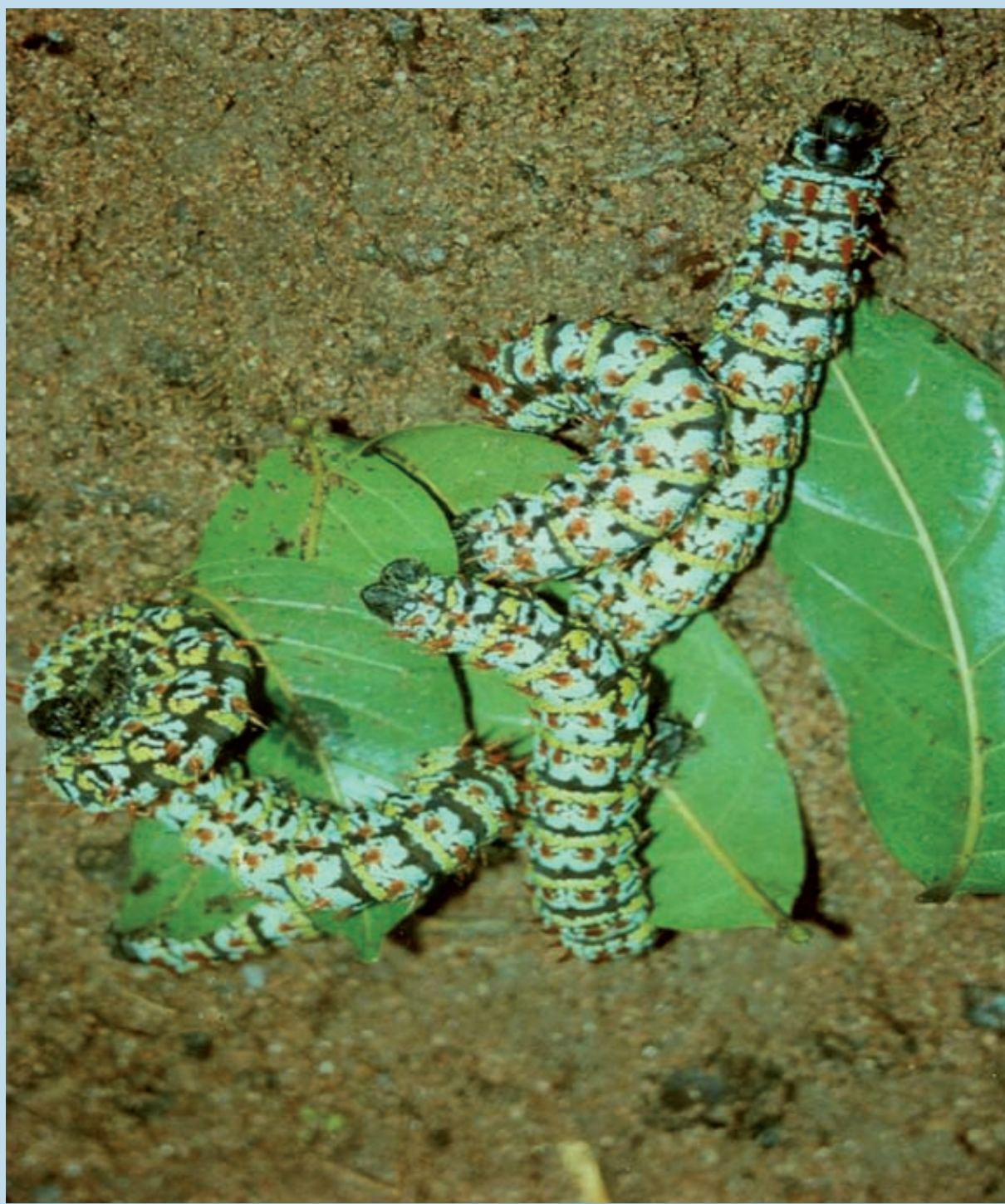




\section{RÉSUMÉ}

\section{LE DÉFI DE LA SYLVICULTURE EN FAVEUR DES PRODUITS FORESTIERS NON LIGNEUX SOUS LES TROPIQUES : DE LA CUEILLETTE À L’AGRICULTURE ?}

Pour passer en revue la sylviculture destinée à favoriser les produits forestiers non ligneux (Pfnl), il faut avant tout bien comprendre et utiliser des définitions et des termes clairs afin d'éviter toute confusion pour les lecteurs et les utilisateurs quant aux « techniques sylvicoles » décrites, à savoir si elles font actuellement partie de la « foresterie » ou du « secteur agricole ». La production sylvicole des Pfnl entre à la fois dans le domaine de la «foresterie » et de l'« agriculture » du fait que la majorité des espèces de Pfnl sont actuellement prises dans un processus dynamique de domestication qui remplace progressivement les pratiques traditionnelles de récolte et de chasse en forêt, pour aller vers des cultures plus intensives d'exploitation agricole. Les interventions sylvicoles qui favorisent le développement des Pfnl au sein des forêts tropicales sont assujetties aux nouvelles perspectives des utilisateurs, qui peuvent aller de la satisfaction des besoins de subsistance jusqu'à la production de produits de base en vue d'une transformation industrielle et d'une commercialisation internationale. Cet article propose une approche descriptive de la complexité des différentes interventions sylvicoles, qui ont pour objectif de gérer les forêts pour produire non seulement des biens et services ligneux mais aussi des Pfnl. La planification des interventions sylvicoles en faveur des Pfnl, en vue de la gestion des forêts, est encore rarement pratiquée. Elle exige une approche intersectorielle pour intégrer les nombreuses demandes, souvent conflictuelles, des utilisateurs sur le plan tant alimentaire que des besoins en bois d'œuvre, bois énergétique et Pfnl. En outre, s'agissant des activités récréatives et de loisirs, elle implique de multiples acteurs qui ne sont pas uniquement concernés par l'exploitation du bois. Les organisations internationales et non gouvernementales jouent un rôle essentiel pour sensibiliser et renforcer les capacités techniques et institutionnelles des pays et faire en sorte qu'ils intègrent la sylviculture des Pfnl dans la gestion durable des forêts.

Mots-clés : açai, gomme arabique, rotin, sapelli, chenilles, produits forestiers non ligneux, plantations, certification, sylviculture tropicale.

\section{ABSTRACT}

\section{FROM GATHERING TO FARMING: THE CHALLENGE OF SYLVICULTURE FOR NON-WOOD FOREST PRODUCTS IN THE TROPICS}

To conduct a review of sylviculture for NonWood Forest Products (NWFP), it is very important to understand and use clearly defined terms in order to avoid confusion among readers and users as to the "sylvicultural techniques" described: in other words, do they currently refer to "forestry" or to the "agricultural sector"? Sylvicultural production of NWFPs comes under both "forestry" and "agriculture", since most NWFP species today are being used in a dynamic domestication process that is gradually replacing traditional hunting and gathering in forests and moving towards more intensive farming practices. Sylvicultural interventions that promote NWFP development in tropical forests are being influenced by new user expectations that range from the satisfaction of subsistence needs to the production of raw materials for industrial processing and international marketing. This article sets out to describe the complexity of the different sylvicultural interventions that aim to manage forests to produce not only timber products and services but also NWFPs. Planned sylvicultural interventions to manage forests for NWFPs are still very rare. They demand an inter-sectoral approach that can handle the many and often conflicting user demands, whether for food, construction timber, fuelwood or NWFPs. Furthermore, where recreational and leisure activities are involved, these demands concern many different players with interests other than forestry. The role of international and non-governmental organisations is essential role to raise awareness and build technical and institutional capacities in the countries concerned, to help them integrate NWFP sylviculture as part of sustainable forest management.

Keywords: acai berries, gum Arabica, rattan, sapelli, caterpillars, non-wood forest products, plantations, certification, tropical sylviculture.
RESUMEN

\section{EL RETO DE LA SILVICULTURA A FAVOR DE LOS PRODUCTOS FORESTALES NO MADEREROS EN LOS TRÓPICOS: DE LA RECOLECCIÓN A LA AGRICULTURA}

Si se quiere analizar la silvicultura destinada a favorecer los productos forestales no maderables (PFNM), primero hay que aclarar el significado y el uso de definiciones y términos para evitar posibles confusiones entre lectores y usuarios en cuanto a las "técnicas silvícolas" descritas. Es decir, ¿forman parte actualmente de la "actividad forestal" o de la "actividad agrícola"? La producción silvícola de los PFNM entra a la vez en el ámbito de lo forestal y de lo agrícola debido a que la mayoría de especies de PFNM se encuentran actualmente en un proceso dinámico de domesticación que sustituye progresivamente las prácticas tradiciones de recolección y caza en el bosque para orientarse hacia cultivos más intensivos de explotación agrícola. Las intervenciones silvícolas que favorecen el desarrollo de los PFNM en los bosques tropicales están sometidas a las nuevas perspectivas de los usuarios, que pueden ir desde la satisfacción de las necesidades de subsistencia hasta la producción de productos de base con miras a una transformación industrial y una comercialización internacional. Este artículo propone un enfoque descriptivo de la complejidad de las distintas intervenciones silvícolas cuyo objetivo es el manejo de bosques para producir no sólo bienes y servicios madereros, sino también PFNM. La planificación de las intervenciones silvícolas de PFNM para lograr una ordenación forestal es aún escasa. Esta planificación requiere un enfoque intersectorial que integre las numerosas demandas, a menudo contrapuestas, de los usuarios, tanto a nivel alimentario como para las necesidades de madera, leña y PFNM. Además, en las actividades recreativas y de ocio, involucra a múltiples actores cuyos intereses van más allá del aprovechamiento maderero. Las organizaciones internacionales y no gubernamentales desempeñan un papel esencial para sensibilizar y reforzar las capacidades técnicas e institucionales de los países e intentar que éstos integren la silvicultura de los PFNM en el manejo sostenible de los bosques.

Palabras clave: asaí, goma arábiga, ratán, sapelli, orugas, productos forestales no madereros, certificación, silvicultura tropical. 


\section{Clarification des termes et définitions liés aux Pfnl}

De nombreux termes sont utilisés pour rendre compte du large éventail de plantes et d'animaux d'origine forestière qui procurent des biens (autres que ligneux) et des services : produits forestiers mineurs, produits forestiers non ligneux, produits secondaires de la forêt... (BELCHER, 2003) et les débats sur les termes et définitions ne sont pas encore clos. Dans ce qui suit, le terme produit forestier non ligneux (Pfnl) est utilisé et défini en tant que produit d'origine biologique autre que le bois dérivé des forêts, d'autres terres boisées et d'arbres hors forêt (FAO, 2000 b). Il est important de noter que cette définition n'inclut pas le bois de feu, les pieux/perches ou l'artisanat en bois et que les activités sylvicoles de leur production ne seront pas évoquées. Notons que les Pfnl peuvent aussi provenir de la collecte ou de la chasse pratiquées par certaines populations aux alentours des forêts ou même en dehors, tels que le miel, les champignons, le gibier ou les noix et châtaignes collectées sur des terres agricoles.

\section{Les dynamiques de production de Pfnl dans les tropiques : de la chasse/cueillette à l'agriculture}

Les forêts tropicales constituent plus de $47 \%$ de la superficie totale mondiale des forêts, avec une superficie estimée à près de 4 milliards d'hectares (ha) par la $\mathrm{FaO}^{1}$ (FAO, 2000 a). Depuis des temps immémoriaux, les populations récoltent toute sorte de végétaux et chassent des animaux dans la forêt pour se nourrir, fabriquer leur maison ou satisfaire leurs besoins énergétiques. Il s'agit de noix comestibles, champignons, fruits, herbes, épices, gommes, plantes aromatiques, gibier, bois, fourrage et produits végétaux ou animaux aux usages médicinaux, cosmétiques, alimentaires ou culturels. Encore aujourd'hui, 1,2 milliard de personnes, avant tout les populations rurales et pauvres des pays en développement, tirent en grande partie leurs ressources et revenus des plantes et animaux issus des forêts (FAO, 2007, 2009). En Inde, par exemple, la Banque mondiale estime que 275 millions de ruraux pauvres dépendent des forêts en termes de moyens d'existence (BANQUE MONDIALE, 2006). Certains des $\mathrm{Pfnl}$ peuvent même être commercialisés à l'échelle nationale et internationale, comme la résine de pin, le rotin, le bambou, les plantes médicinales et la gomme arabique. L'utilisation des Pfnl relève du secteur informel ; c'est pourquoi peu de données fiables sont disponibles en ce qui concerne leur production, leur commerce et le nombre de personnes impliquées dans ce secteur.

À travers le monde, $90 \%$ de la production totale rapportée par pays provient de cinq principales catégories d'usages de Pfnl, soit par ordre d'importance décroissant : les « aliments » avec comme principaux produits les fruits, les baies, les champignons et les noix ; les " exsudats",

\footnotetext{
${ }^{1}$ Food and Agriculture Organization of the United Nations.
}

avant tout la gomme arabique et les résines; les « autres produits végétaux » tels que le bambou, le rotin et le liège ; le « miel » et les «plantes décoratives » dont font surtout partie les arbres de Noël.

Les principaux pays producteurs de Pfnl tropicaux sont la Chine, l'Inde, l'Indonésie et le Brésil (FAo, 2005). Au moins 150 sortes de Pfnl sont importantes pour le commerce international. De plus, un grand nombre de plantes (approximativement 4000 espèces) sont commercialisées sur les marchés internationaux. La majorité de ces produits sont plutôt vendus en petites quantités, bien que certains comme les racines de ginseng, le miel naturel et les noix et pignons, le colophon, le rotin et la gomme arabique soient commercialisés à des niveaux substantiels (FAO, 1995).

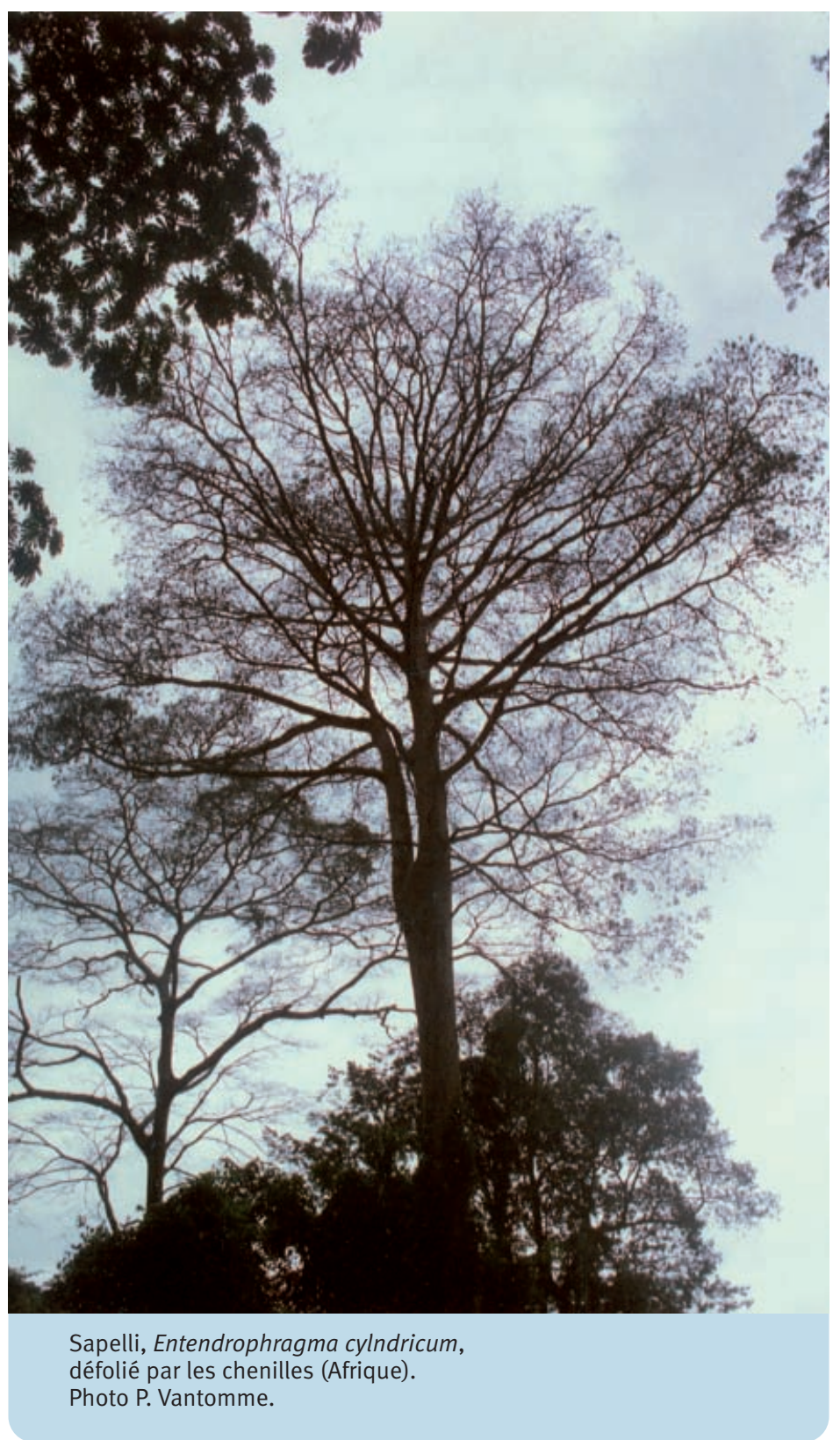




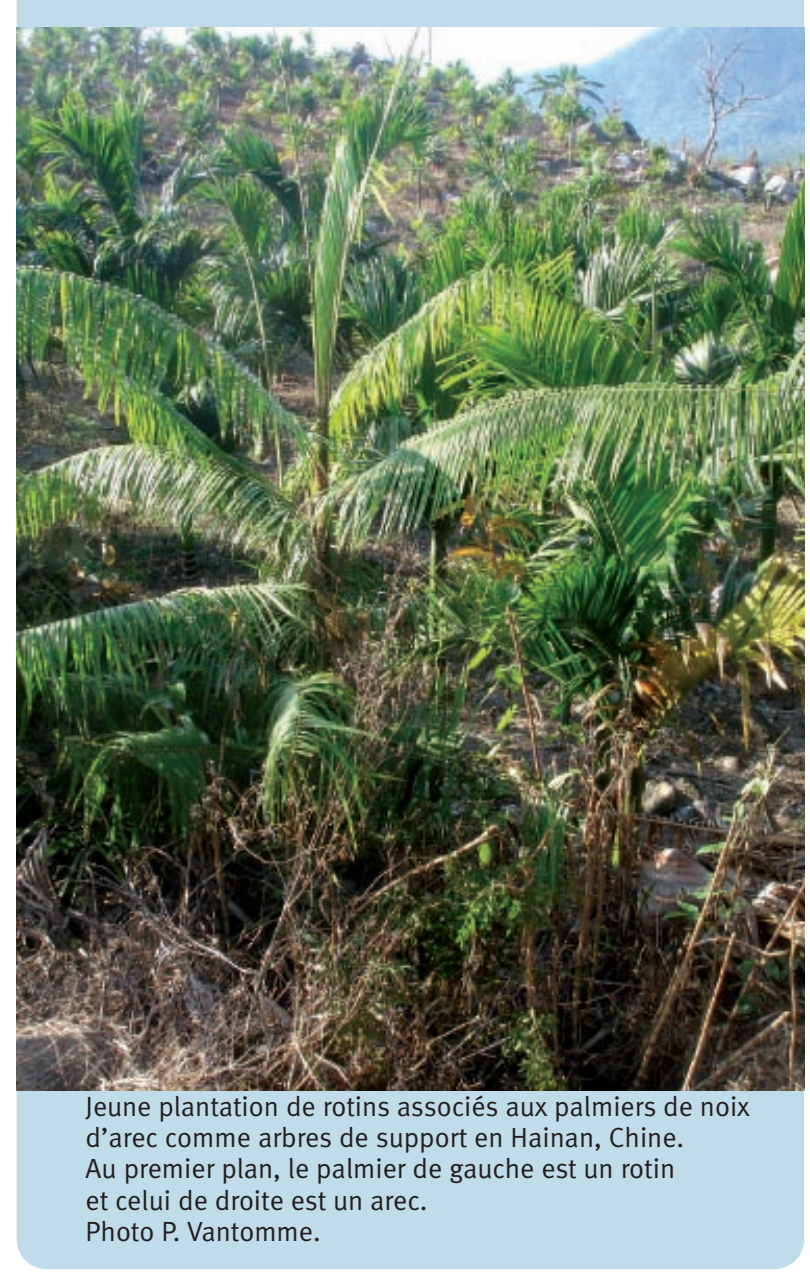

Les principaux Pfnl produits par région (FA0, 2000 a) sont les suivants :

- Europe : aliments, miel, produits décoratifs et de la chasse ; la Russie, l'Allemagne, l'Espagne, le Portugal et l'Italie sont les principaux pays producteurs ;

- Asie : aliments, autres produits végétaux et exsudats ; la Chine est de loin le plus grand producteur asiatique, suivie parl'Inde ;

- Afrique : exsudats et aliments ; le Soudan (gomme arabique) et l'Afrique du Sud (plantes médicinales) sont considérés comme les principaux producteurs de la région ;

- Amérique du Nord et du Sud : les autres produits végétaux, aliments et exsudats sont les principales catégories, provenant avant tout des États-Unis, du Canada, du Brésil et de la Colombie :

- Océanie : aliments et matières premières pour la construction constituent les principaux Pfnl.

En fait, il faut bien avoir à l'esprit que la majorité des espèces cultivées actuellement et des animaux domestiques qui accompagnent les populations dans leur vie quotidienne proviennent d'espèces qui poussaient ou vivaient auparavant à l'état sauvage et étaient récoltées ou chassées par l'homme de manière traditionnelle. Graduellement, au cours de l'histoire humaine et du développement de l'agriculture, ces plantes et animaux ont fourni les sources de matériel génétique pour les programmes de domestication (telles les cultures tropicales comme le café, le cacao, l'huile de palme, l'hévéa, le thé, la vanille, la girofle, le bambou, l'ananas, les mangues, etc.). Ce processus de domestication des plantes et des animaux sauvages issus des forêts tropicales est encore d'actualité. Parmi les espèces forestières tropicales bien connues et récemment domestiquées, il est possible de citer les noix de macadamia, Macadamia integrifolia, le fruit du carambolier, Averrhoa carambol, le cupuacu, Theobroma grandifolia, et le durian, Durio zibethinus, mais aussi les espèces animales: le paca, Agouti paca, ou les iguanes, Iguana iguana.

Sous les tropiques, les Pfnl proviennent de toute une série de systèmes de production allant de la simple collecte des produits à l'état sauvage, dans ou hors la forêt, par les populations autochtones - comme c'est le cas des noix du Brésil en Amazonie avec une production annuelle moyenne d'environ 35000 tonnes (VANTOMME, 2004) - à des formes beaucoup plus intensives de domestication et d'intensification des cultures grâce à l'agroforesterie, l'agriculture ou même de vastes plantations industrielles dont celles de bambou moso, Phyllostachys pubescens, qui s'étendent sur 2,7 millions d'hectares en Chine (Ruiz PÉREz et al., 1999). Il en est de même pour l'hévéa, Hevea brasiliensis, au Brésil, dont le latex est encore récolté par les populations locales d'Amazonie en forêt alors qu'en parallèle, dans le même pays, des plantations industrielles de clones améliorés d’hévéas sont développées suivant des modèles industriels. Le même scénario s'observe pour l'huile de palme au Cameroun ou le durian en Malaisie. Ces différents systèmes de production regroupent de nombreux acteurs qui produisent suivant différents modes sylvicoles à différents niveaux d'intensité. Si gérer les espèces de Pfnl dans les forêts peut faire partie de la sylviculture, pour leur part, les systèmes de production plus intensifs, qu'ils soient agricoles ou de plantation, relèvent des sciences agricoles et horticoles.

\section{La sylviculture des principaux Pfnl tropicaux}

Afin d'illustrer ce qui précède, plusieurs approches sylvicoles majeures de production de Pfnl sont présentées pour ce qui est des forêts tropicales du Bassin amazonien mais aussi d'Afrique et d'Asie.

L'açaí, du nom local auquel appartiennent les espèces Euterpe oleracea, E. precatoria et d'autres Euterpe spp., fait partie des Pfnl les plus connus des forêts amazoniennes. Les espèces de ce palmier à plusieurs tiges sont très répandues dans les plaines inondables et sont avant tout utilisées pour la récolte des cœurs de palmier (palmito) et des fruits comestibles. Ces palmiers peuvent atteindre plus de $20 \mathrm{~m}$ de hauteur et $40 \mathrm{~cm}$ de diamètre. Ils poussent de façon grégaire avec souvent plus de 100 adultes par hectare. Les fruits viennent à maturité entre septembre et décembre, avec une dispersion ultérieure des graines par les oiseaux et les rongeurs (Kageyama et al., 2004 ; RochA, VIANA, 2004). L'abondance et la croissance rapide d'Euterpe oleracea étaient déjà appréciées par les populations précolombiennes qui entretenaient ces arbres tout en se limitant à la collecte sur les peuplements sauvages, associée à certaines cultures de subsistance près de leurs champs. Ce système d'extraction n'a subi aucun bouleversement jusqu'à approximativement les années 1970. 
Depuis, la demande commerciale pour les cœurs de palmier açaí et pour le jus qui est extrait de ses fruits s'est accrue, de même que les forêts naturelles des plaines inondables sont devenues plus accessibles en raison de l'exploitation, pour son bois, du virola (Virola surinamensis et Virola. spp.) en vue d'alimenter l'industrie en plein essor du contreplaqué en Amazonie. L'exploitation du bois dans les plaines inondables d'Amazonie a permis l'expansion de l'industrie des cœurs de palmier en conserve, car, d'après les témoignages recueillis, l'exploitation de Virola dans les forêts naturelles inondables aurait amélioré les conditions de croissance des palmiers açaí par la pénétration plus forte de la lumière dans la forêt. D'une simple collecte des peuplements naturels d'accès libre, l'approche sylvicole s'est progressivement développée en un système de rotation de coupe imposé par les compagnies forestières, ce qui a permis à ces forêts exploitées de se régénérer (TAYLOR, 2005). Malgré tout, même si les plantations d'açaí de génotypes améliorés émergent, le système d'extraction des peuplements naturels fournit encore la majorité de la production des cœurs de palmier et des fruits, du simple fait que la récolte des peuplements naturels coûte moins cher que de développer des plantations.

La gomme arabique est une gomme naturelle récoltée par entaille de l'écorce des acacias notamment (Acacia senegal et Acacia seyal). La gomme récoltée est issue de l'exploitation des acacias à l'état sauvage ou dans les plantations d'acacias dans tout le Sahel, du Sénégal à la Somalie. L'acacia est très répandu dans les savanes sèches d'Afrique et les arbres se régénèrent facilement (FAGG, ALLISON, 2004). Traditionnellement, les pasteurs récoltaient la gomme arabique sur les acacias qu'ils rencontraient sur leur passage lors de la saison de transhumance. Dans certaines régions du Soudan, qui est le plus gros producteur de gomme arabique au monde (BANQUE MONDIALE, 2007), les fermiers pratiquent la culture sur brûlis et tolèrent la régénération spontanée de quelques acacias (approximativement un semis tous les 10 mètres). Après deux ou trois années d'exploitation agricole, la fertilité des sols baissant, les parcelles sont abandonnées pour cultiver de nouvelles terres; des jeunes plants poussent alors rapidement et forment un peuplement d'acacias ouvert en quatre à cinq années (WorLd Agroforestry CENTER, 2010 ; RAHIM et al., 2005). Pendant cette période, le pâturage dans ces jeunes peuplements d'acacias est interdit. Les bergers saisonniers qui migrent avec leurs animaux peuvent ensuite faire paître leurs bêtes durant 15 à 20 années, suivant la disponibilité des terres et les conditions atmosphériques. Ce système sylvicole est un bon exemple de symbiose entre agriculteurs et bergers, pour faire pousser des arbres sur des parcelles de terres agricoles, qui peuvent ainsi restaurer la fertilité des sols et fournir en même temps du fourrage, de la gomme arabique, du bois de feu et du charbon de bois aux populations (BoFFA, 1999).

En Afrique centrale, le cas des chenilles récoltées sur le sapelli, Entandrophragma cylindricum, s'avère fort intéressant. La Fao a commissionné des études de cas en 2004 auprès d'experts nationaux au Cameroun, en République centrafricaine, République du Congo (Brazzaville) et République démocratique du Congo pour documenter le rôle des insectes comestibles récoltés en forêt au sein des pays respectifs et la relation entre la récolte des chenilles et la gestion des forêts. Ces études se sont avant tout intéressées aux chenilles de sapelli car elles sont très répandues dans les forêts, peuvent se récolter très facilement mais aussi constituent une source d'alimentation abondante et populaire en Afrique centrale. La majorité des chenilles se nourrissent des feuilles des arbres y compris des feuilles d'espèces importantes pour le bois d'œuvre comme le sapelli. Leur impact sur la santé des arbres et sur l'écosystème forestier en général est aussi assez significatif pour analyser les nombreuses options de gestion des forêts possibles, afin de satisfaire à la fois aux besoins locaux en nourriture mais aussi à la santé et à la productivité des écosystèmes forestiers. L'exploitation des grumes de sapelli constitue en fait un cas particulier où la survie des insectes comestibles peut être menacée par l'élimination de son arbre hôte (FAO, 2004).

En République centrafricaine, les règlements actuels des concessions forestières stipulent l'obligation pour les exploitants de régénérer un certain nombre de sapellis par hectare de forêt exploité. La logique de cette mesure est purement sylvicole, pour permettre la régénération naturelle, et ne prend pas en compte l'engouement des populations locales vis-àvis des espèces de chenille (Imbrasia oyemens) qui se nourrissent notamment des feuilles de sapelli. Ces chenilles sont très appréciées en tant que mets délicieux et surtout très nourrissant, pouvant être consommé en famille ou vendu sur le marché. Étant donné qu'il est plus facile de récolter des chenilles en coupant les arbres de faible diamètre, cette pratique de cueillette met en péril la régénération du sapelli dans les concessions forestières. Pour éviter les conflits entre exploitants forestiers et populations locales, il est possible d'envisager de planter des sapellis plus proches des habitations et des cultures des paysans, évitant que les populations n'aillent dans les concessions forestières couper des jeunes arbres d'avenir pour récolter les chenilles (VANTOMME et al., 2004). Il est aussi possible d'imaginer une exploitation des

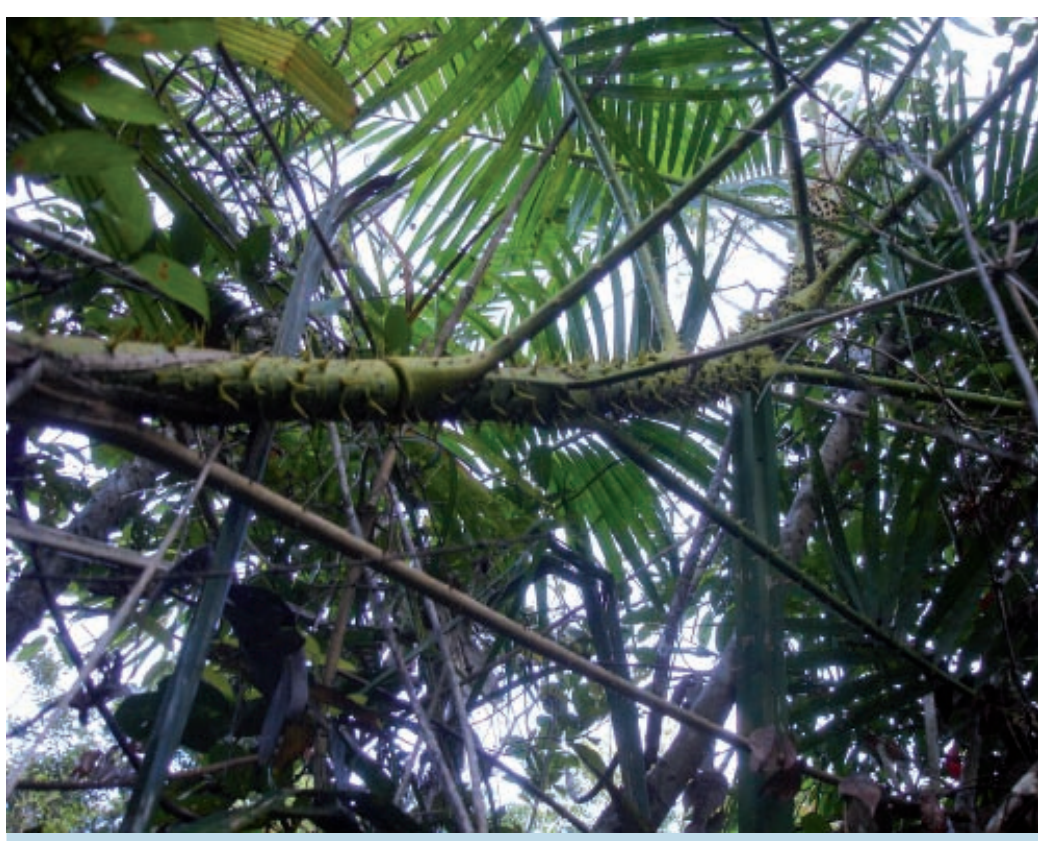

Tiges de rotin recouvertes d'épines illustrant la difficulté de travailler le rotin. Photo P. Vantomme. 


\section{Opportunités et défis en matière d'introduction de la sylviculture des Pfnl tropicaux}

chenilles sur des arbres taillés en têtard, cette technique de taille devant permettre aux populations de couper plus facilement une partie des branches pour ramasser les chenilles qui recouvrent les feuilles.

En Asie tropicale, le rotin, notamment Calamus manan, est un Pfnl très répandu. Les rotins sont des tiges de palmier qui poussent comme des lianes dans les forêts humides tropicales. Il existe treize genres différents de rotin qui rassemblent environ 600 espèces (DRANSFIELD, 2002). Le rotin, une fois transformé, a de multiples usages : réalisation de paniers, filets ou cordages mais aussi fabrication de meubles. Ces palmiers-rotin se trouvaient facilement en forêt, mais les niveaux d'exploitation actuels élevés (guère durables) ainsi que la déforestation en ont réduit significativement les réserves. La réponse de la sylviculture à la réduction de l'offre en rotin a donc été double : d'une part, par la mise en place d'une meilleure protection du rotin à l'état sauvage dans les forêts naturelles et, d'autre part, par la création de plantations (SASTRY, 2002). Le rotin a tout d'abord été inclus dans les activités de gestion des forêts naturelles faisant intervenir la sylviculture pour promouvoir leur croissance (en éclaircissant les bouquets lorsqu'ils sont trop denses et en ne laissant que quelques tiges par bouquet/touffe) et en imposant des licences pour leur exploitation. À titre d'exemple, les inventaires forestiers cartographient maintenant les stocks de rotin par espèce et par taille (nombre de tiges par bouquet) et l'exploitation des tiges matures est prévue avec un cycle d'environ quinze ans. Le plan de gestion des forêts laisse des arbres de support pour permettre aux tiges de rotin de grimper à la recherche de la lumière. Et lorsque cela est nécessaire, certaines forêts sont enrichies en rotin (en particulier avec des espèces qui fournissent des cannes de large diamètre) (TESORO, 2002).

Les plantations de rotin sur des terres forestières défrichées ont principalement été essayées en Malaisie, aux Philippines et en Indonésie, en combinaison éventuelle avec la plantation d'hévéas comme arbres de support. L'association hévéa-rotin a été envisagée depuis le début des années 1990. Ces essais ont montré que le rotin ne peut être planté qu'en fin de cycle de l'hévéa, à partir de la vingt-cinquième année, et non au début car la récolte du rotin est extrêmement dommageable pour les hévéas (PenOt, OlLIVIER, 2009). Toutefois, la complexité à développer des plantations de rotin pose encore de nombreux défis à la recherche, tant au plan technique que sylvicole; par exemple : la sélection des arbres de support les plus adaptés; comment raccourcir les cinq à sept années de stade en rosette dans lequel les jeunes palmiers plantés ne développent pas de tiges ; comment améliorer la récolte, très laborieuse, des tiges de rotin qui peuvent mesurer de 20 à 50 mètres de long et être recouvertes de nombreuses épines. Il faut aussi souligner que les populations rurales ne sont pas toujours attirées par le travail des plantations de rotin, qui leur rapporte moins que les cultures d'huile de palme ou d'hévéa. Aménager le rotin dans les forêts naturelles reste donc encore la meilleure option pour produire des tiges de rotin (RAZAK MOHD, RAJA BARIZAN, 2001 ; FAO, 2002).
L'intérêt d'inclure les Pfnl dans la gestion des forêts est maintenant reconnu par diverses conventions internationales telles que la Convention sur la diversité biologique ( $\mathrm{Cdb}$ ), les initiatives sur les forêts, les critères et indicateurs pour la gestion durable des forêts (Gdf), ou les programmes forestiers nationaux de nombreux pays. En outre, les gouvernements, les organismes de développement, et en particulier, les Ong ont de fortes attentes vis-à-vis d'une meilleure utilisation possible des Pfnl en vue de favoriser la conservation de la biodiversité et les programmes de sécurité alimentaire et de subvenir aux besoins des populations dépendantes des forêts. Depuis les années 1990, l'importance accordée aux composantes environnementales et sociales de la Gdf s'est fortement accrue et a fait apparaître la nécessité d'incorporer les objectifs liés aux Pfnl dans la gestion sylvicole des forêts tropicales et en particulier dans les accords de concessions forestières dans le processus de leur certification. Cependant, font encore défaut de nombreuses informations de base sur la dynamique des populations des principales espèces tropicales de Pfnl, sur leurs besoins sylvicoles et leurs prescriptions de récolte. Même si les producteurs et les collecteurs possèdent des connaissances ancestrales sur la gestion des différentes espèces récoltées dans leurs forêts pour leur subsistance, il s'avère qu'aujourd'hui ces connaissances ne suffisent plus pour répondre à la demande commerciale croissante et/ou aux changements en matière de préférences des consommateurs concernant la qualité des produits.

Les contraintes et les défis de la sylviculture appliqués aux forêts tropicales, avec pour objectif d'accroître la production des Pfnl, peuvent être résumés comme suit.

Dans les forêts naturelles tropicales, la faisabilité technique du recours à la sylviculture pour développer la production de bois tout en soutenant l'offre d'espèces de Pfnl

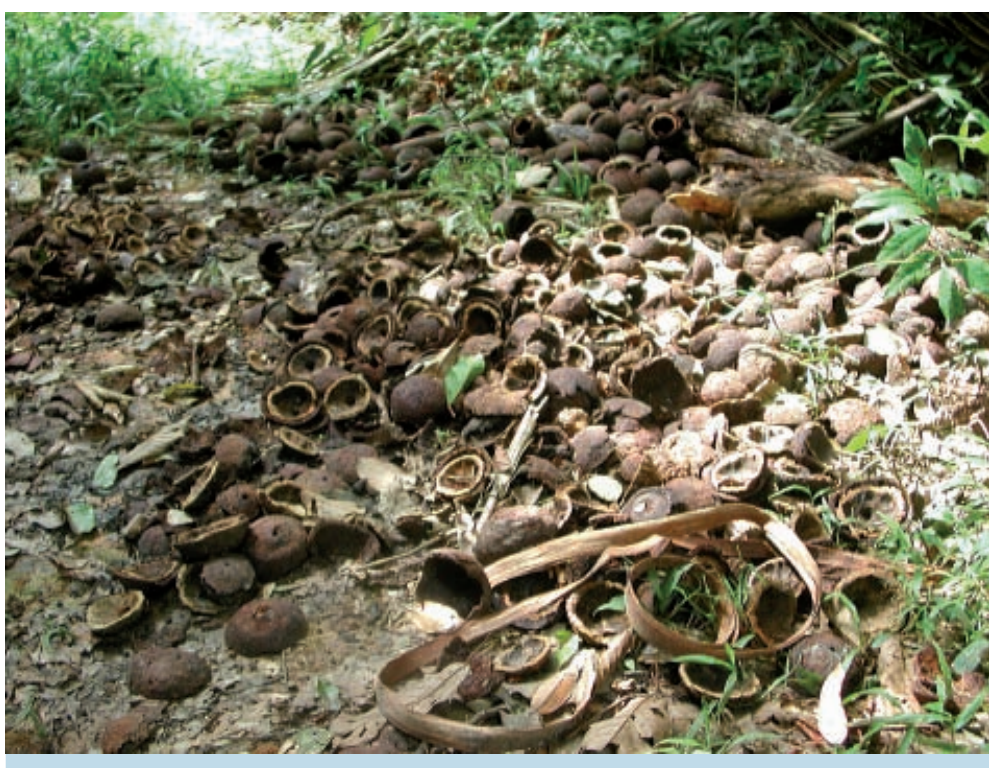

Résidus de coques abandonnés en forêt après décortication des noix du Brésil pour leur commercialisation en ville. Photo P. Vantomme. 
commerciales est très complexe du fait qu'il existe de très nombreuses espèces et formes de vie possibles : feuilles, racines, écorce, fruits, noix... Les interventions sylvicoles peuvent aussi être, dans de nombreux cas, conflictuelles entre elles. Par exemple, les arbres qui produisent des noix, de l'écorce, des fruits, des feuilles ou des chenilles comestibles ont besoin d'espace dans la canopée supérieure, avec une pleine exposition à la lumière du soleil et de multiples coupes pour stimuler le développement d'un large houppier à faible hauteur, facilitant la récolte des Pfnl. La sylviculture pour la production de bois d'œuvre est essentiellement opposée à ce principe, l'objectif étant de faire croître de jeunes arbres à forte densité, progressivement éclaircis, pour obtenir des fûts de qualité. Les différents acteurs peuvent aussi avoir des intérêts divergents. Les concessionnaires forestiers d'Afrique centrale veulent, par exemple, exploiter le sapelli pour son bois de grande valeur, alors que les populations locales recherchent cet arbre pour y récolter les chenilles qui se nourrissent de ses feuilles (VANTOMmE et al., 2004). D'autre part, lorsque la valeur commerciale d'un Pfnl donné augmente fortement comme la cannelle (issue de l'écorce interne du cannelier, Cinnamomum verum), les autres arbres seront progressivement coupés et remplacés par des canneliers, transformant la forêt naturelle en un « verger de canneliers " de propriétaires privés (MıCHON et al., 1986). Dans la majorité des cas, la domestication des espèces de Pfnl a souvent eu pour conséquence de remplacer la forêt originelle par des plantations de Pfnl (avec privatisation des terres, formelle ou informelle), comme par exemple la production de bambous en Chine, ou de la cardamome ou de la girofle en Inde, laissant bien peu de place à la biodiversité auparavant présente dans ces forêts.

Le commerce mondial des Pfnl a intensifié la compétition pour accroître la productivité et la qualité des produits. Les Pfnl commercialisés proviennent de plus en plus de systèmes de production agricole rendant les produits de cueillette moins compétitifs et/ou de moindre qualité. La domestication et la production de Pfnl sur des terres agricoles réduisent la valeur économique de ces mêmes espèces qui poussent spontanément dans les forêts. Pour la plupart des Pfnl commerciaux, les récolteurs tendent à devenir des acteurs marginalisés dès lors que les volumes d'échanges commerciaux s'accroissent et que la production s'intensifie ; et en règle générale, les bénéfices vont plutôt aux commerçants et aux agriculteurs.

Les systèmes de certification pour la gestion durable des forêts ou pour le commerce équitable peuvent offrir, certes à un coût élevé, un certain degré de protection de la production auprès des petits récolteurs forestiers de zones certifiées. Toutefois, les bénéfices de la certification ne sont pas faciles à partager auprès de tous les producteurs du secteur (VANTOMme, WALter, 2003). Il est donc primordial de contrôler l'impact social de la domestication des Pfnl sur les conditions de vie des collecteurs de ces produits. Alors que la certification des forêts et de leurs bois progresse nettement grâce aux différents systèmes ayant été mis en place, le développement de critères et standards techniques et sociaux pour la certification des Pfnl en est encore à ses débuts. Au plan commercial, la certification des Pfnl rencontre des écueils en ayant pour but d'atteindre les marchés internationaux, puisque les $\mathrm{Pfnl}$ sont vendus principalement sur les marchés locaux et nationaux. Même si les échanges commerciaux de produits forestiers non ligneux certifiés sont en nette croissance, ils n'en restent pas moins de faible envergure.

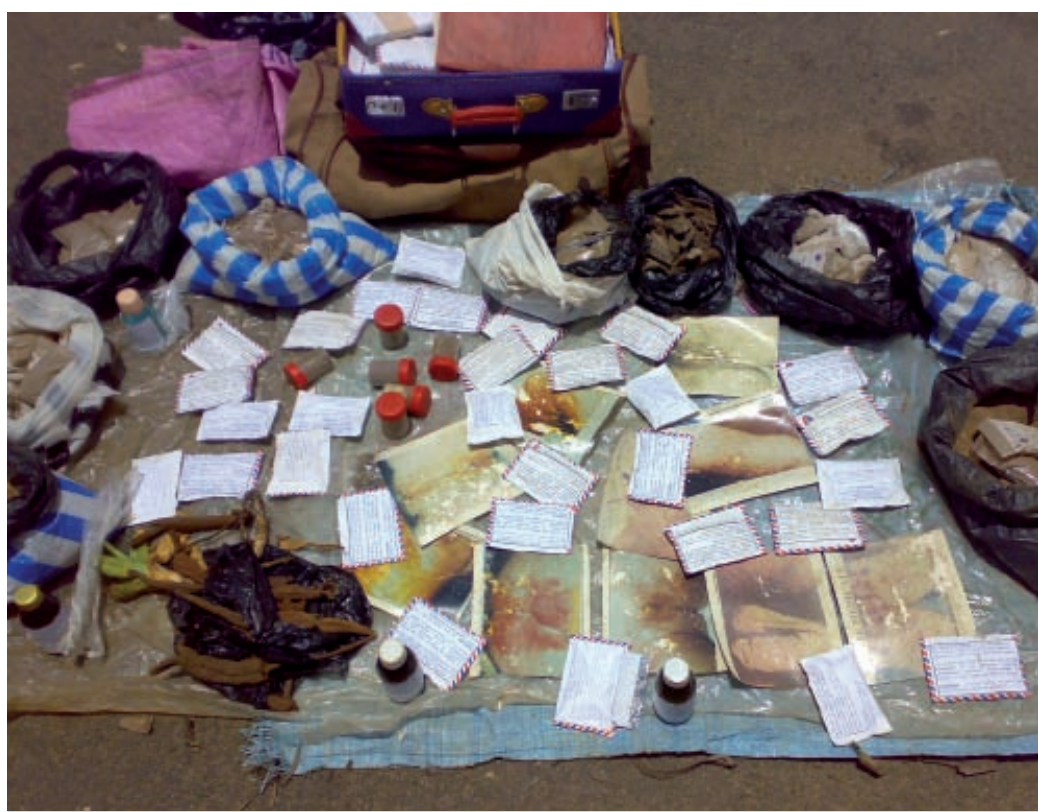

Plantes médicinales et remèdes vendus par un guérisseur sur un marché du Cameroun.

Photo P. Vantomme.

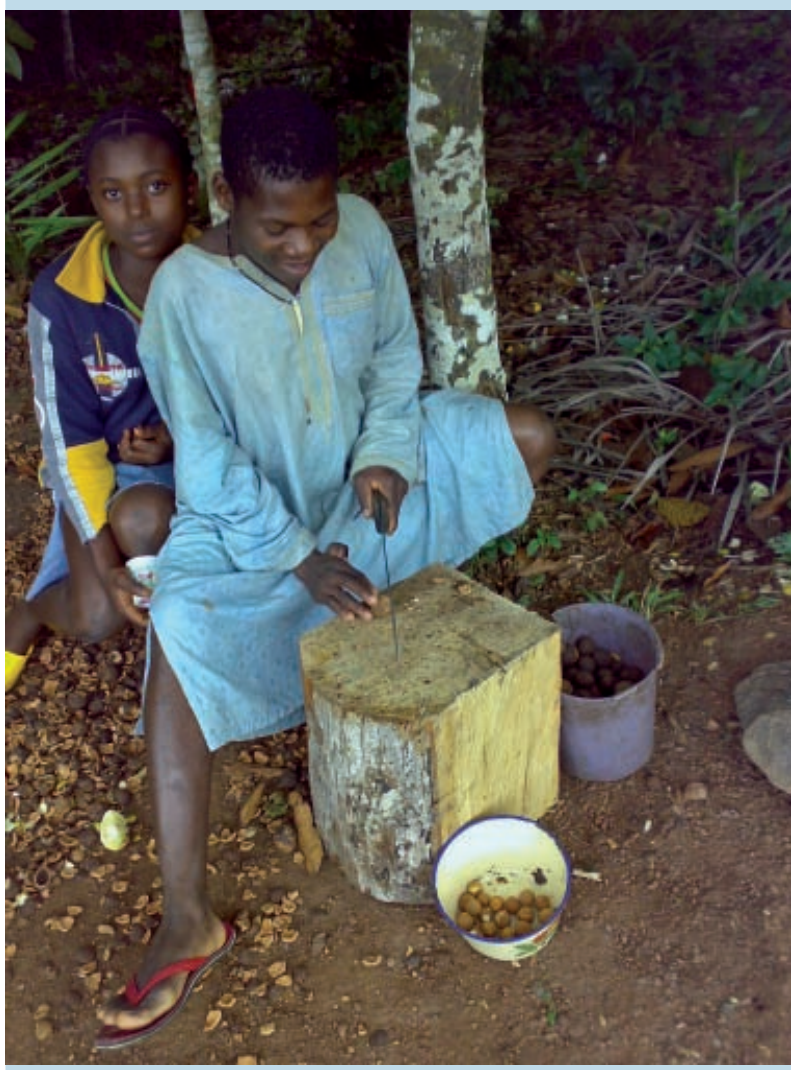

Jeune garçon décortiquant une noix de cola (Coula edulis) au Cameroun.

Photo P. Vantomme. 


\section{Conclusion}

Pour passer en revue la sylviculture des produits forestiers non ligneux, il faut avant tout bien comprendre et utiliser des définitions et des termes clairs afin d'éviter toute confusion pour les lecteurs s'agissant des techniques "sylvicoles» décrites. Le terme produit forestier non ligneux (Pfnl) est utilisé et défini en tant que produit d'origine biologique autre que le bois dérivé des forêts, d'autres terres boisées et d'arbres hors forêt.

Depuis des temps immémoriaux, les populations récoltent toute sorte de végétaux et chassent des animaux dans la forêt pour se nourrir, fabriquer leur maison ou satisfaire leurs besoins énergétiques. Encore aujourd'hui, 1,2 milliard de personnes, avant tout les populations rurales et pauvres des pays en développement, tirent une grande partie de leurs besoins de subsistance et revenus des plantes et animaux issus des forêts. L'utilisation des Pfnl fait partie essentiellement du secteur informel. C'est pourquoi peu de données fiables sont disponibles en ce qui concerne la production, le commerce et le nombre de personnes impliquées dans ce secteur.

La majorité des espèces cultivées actuellement proviennent d'espèces qui poussaient auparavant à l'état sauvage et étaient récoltées par l'homme de manière traditionnelle. Graduellement, au cours de l'histoire humaine et du développement de l'agriculture, ces espèces ont fourni le matériel génétique pour leur domestication. Ce processus de domestication des plantes et des animaux sauvages issus des forêts tropicales est encore d'actualité. Les Pfnl tropicaux proviennent de toute une série de systèmes de production allant de la simple collecte des produits à l'état sauvage, dans la forêt ou hors d'elle, par les populations autochtones à des formes beaucoup plus intensives de domestication et d'intensification des cultures grâce à l'agroforesterie, l'agriculture ou même de vastes plantations à l'échelle industrielle. Ces différents systèmes de production regroupent de nombreux acteurs qui produisent suivant différents modes sylvicoles à différents niveaux d'intensité. Si gérer les espèces de Pfnl dans les forêts peut faire partie de la sylviculture, pour leur part, les systèmes de production plus intensifs, qu'ils soient agricoles ou de plantation, relèvent des sciences agricoles et horticoles.

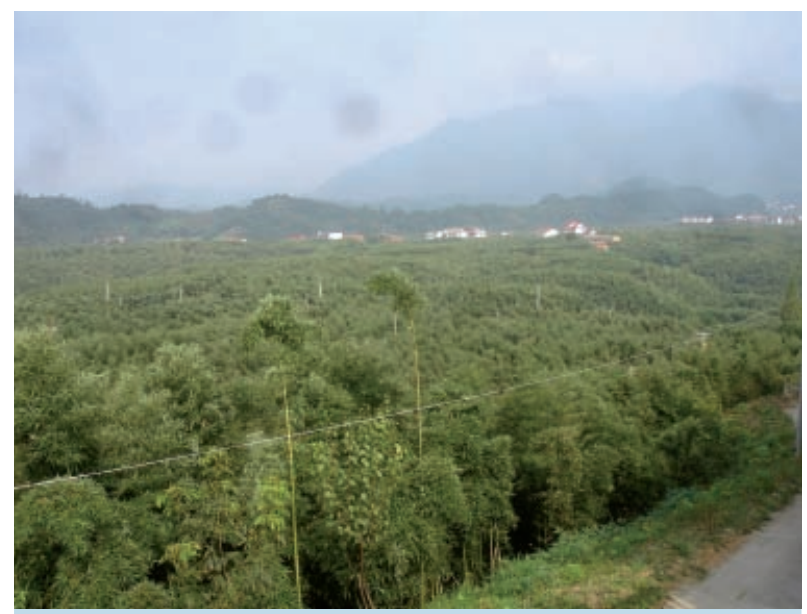

Vallée recouverte de plantations de bambous remplaçant les anciennes cultures de riz moins rémunératrices pour les agriculteurs en Linan, Chine. Photo P. Vantomme.
Quelques approches sylvicoles de production majeures de Pfnl sont présentées, telles que pour l'açaí, la gomme arabique et le rotin. Malgré tout, même si des plantations de ces espèces émergent, le système d'exploitation des peuplements naturels fournit encore la majorité de leur production, du simple fait que la récolte des peuplements naturels coûte moins cher que de développer des plantations.

Depuis les années 1990, l'importance accordée aux composantes environnementales et sociales de la gestion durable des forêts s'est fortement accrue et a fait apparaître la nécessité d'incorporer les objectifs liés aux Pfnl dans la gestion sylvicole des forêts tropicales et en particulier dans les accords de concessions forestières dans le processus de leur certification. Cependant, font encore défaut de nombreuses informations de base sur la dynamique des populations des principales espèces tropicales de Pfnl, sur leurs besoins sylvicoles et leurs prescriptions de récolte. Même si les producteurs et les collecteurs possèdent des connaissances ancestrales sur la gestion des différentes espèces récoltées dans leurs forêts pour leur subsistance, il s'avère qu'aujourd'hui ces connaissances ne suffisent plus pour répondre à la demande commerciale croissante et/ou aux changements en matière de préférences des consommateurs concernant la qualité des produits.

La planification des interventions sylvicoles d'espèces fournissant des Pfnl est encore rarement pratiquée dans le cadre de la gestion des forêts. Celle-ci exige une approche intersectorielle pour intégrer les nombreuses demandes, souvent conflictuelles, des utilisateurs tant au plan alimentaire qu'en ce qui concerne les besoins en fibres, énergétiques et de santé, mais aussi les activités récréatives et de loisirs. Cette planification sylvicole implique ainsi toute une gamme d'acteurs qui ne sont pas seulement concernés par l'exploitation du bois. Les connaissances et les techniques en matière de gestion et de récolte des Pfnl, en particulier les méthodologies pour évaluer leurs impacts sur la durabilité des ressources et sur les moyens d'existence des collecteurs, nécessitent encore de nombreuses améliorations.

Le commerce mondial des Pfnl a intensifié la compétition pour accroître la productivité et la qualité des produits. Les Pfnl commercialisés proviennent de plus en plus de systèmes de production agricole rendant les produits de cueillette moins compétitifs et/ou de moindre qualité. La domestication et la production de Pfnl sur des terres agricoles réduisent la valeur économique de ces mêmes espèces qui poussent spontanément dans les forêts. Pour la plupart des Pfnl commerciaux, les récolteurs tendent à devenir des acteurs marginalisés dès lors que les volumes d'échanges commerciaux s'accroissent et que la production s'intensifie.

Les organisations internationales telles que la Fao peuvent et doivent jouer un rôle fondamental pour sensibiliser et développer les capacités techniques et institutionnelles des pays tropicaux permettant d'intégrer la sylviculture des Pfnl dans des pratiques de gestion durable des forêts, qui soient, sans conteste, équitables en termes sociaux. 


\section{Références bibliographiques}

BANQUE MONDIALE, 2006. Unlocking opportunities for forest-dependent people in India. Grant Milne, WB Report No. 34481-IN, 107 p

BANQUE MONDIALE, 2007. Note politique. Export Marketing of Gum Arabic from Sudan. 24 p. http://siteresources.worldbank.org/INTAFRMDTF/Resources/Gum_Arabic_Policy_Note.pdf BELCHER B., 2003. What isn't a NTFP ? International Forestry Review, 5 (2) : 161-168.

BOFFA J.-M., 1999. Agroforestry parklands in sub-Saharan Africa. Rome, Italie, Fao, Conservation Guide 34, 231 p.

DRANSFIELD J., 2002. General introduction to rattan - the biological background to exploitation and the history of rattan research. In : Rattan : Current research issues and prospects for conservation and sustainable development. Rome, Italie, Fao, Non-Wood Forest Product Series 14, 272 p. http://www.fao.org/ DOCREP/003/Y2783E/y2783e00.htm\#TopOfPage

FAGG C. W., ALLISON G. E., 2004. Acacia senegal and the gum arabic trade. Monograph and annotated bibliography. Oxford, Royaume-Uni, Oxford Forestry Institute, Tropical Forestry Papers 42, $261 \mathrm{p}$.

FAO, 1995. Trade restrictions affecting international trade in non-wood forest products. Rome, Italie, Fao, Non-Wood Forest Product Series 8. http://www.fao.org/docrep/V9631e/ v9631e00.htm

FAO, 2000 a. Global forest resources assessment 2000. Rome, Italie, Fao, p. 326-329. http://www.fao.org/forestry/ fra2000report/en/

FAO, 2000 b. What are non-wood forest products ? 1 p. http://www.fao.org/forestry/site/6388/en

FAO, 2002. Forestry statistics and data collection ASDCA/TN/01 : Non-wood forest products in 15 countries of Tropical Asia: An overview. Rome, Italie, Fao. http://www.fao.org/DOCREP/005/AB598E/AB598E00.HTM

FAO, 2004. Contribution des insectes de la forêt à la sécurité alimentaire. L'exemple des chenilles d'Afrique centrale. Produits forestiers non ligneux. Document de travail $n^{\circ} 1$. Rome, Italie, Fao, Département des forêts. http://www.fao.org/ docrep/007/j3463f/j3463f00.HTM

FAO, 2005. Situation des forêts du monde 2005 - International trade in non-wood forest products. Rome, Italie, Fao, p. 14-19.

FAO, 2007. Situation des forêts du monde 2007. Rome, Italie, Fao. http://www.fao.org/docrep/009/a0773e/ a0773e00.htm

FAO, 2009. Forests and poverty reduction. Rome, Italie, Fao. http://www.fao.org/forestry/livelihoods/en/

KAGEYAMA et al., 2004. Genetic and ecological aspects of nonwood forest product exploitation in two western Amazonian settlements. In : Vinceti B., Amaral W., Meilleur B. (éd.). Challenges in managing forest genetic resources for livelihoods : examples from Argentina and Brazil. Rome, Italie, International Plant Genetic Resources Institute, p. 149-166.
MICHON G., MARY F., BOMPARD J., 1986. Multistoried agroforestry garden system in West Sumatra, Indonesia. Agroforestry Systems, 4 : 315-338.

PENOT E., OLLIVIER I., 2009. L'hévéa en association avec les cultures pérennes, fruitières ou forestières : quelques exemples en Asie, Afrique et Amérique latine. Bois et Forêts des Tropiques, 301 : 67-82.

RAHIM A. H., RUERD R., VAN IERLAND EKKO C., 2005. Adoption and abandonment of gum arabic agroforestry in Sudan. In : Proceedings of the 9th North American Agroforestry Conference. AFTA 2005, Rochester, États-Unis, University of Minnesota.

RAZAK MOHD A., RAJA BARIZAN R. S., 2001. Intercropping rattan with rubber and other crops. Unasylva, 52 (205) : 9.

ROCHA E., VIANA V. M., 2004. Manejo de Euterpe precatoria Mart. (Açaî) no Seringal Caquetá, Acre, Brasil. Scientia Forestalis, 65 : 59-69.

RUIZ PÉREZ M., MAOGONG Z., BELCHER B., CHEN X., MAOYI F., JINZHONG X., 1999. The role of bamboo plantations in rural development : The case of Anji County, Zhejiang, China. World Development, 27 (1) : 101-114.

SASTRY C. B., 2002. Rattan in the twenty-first century - an outlook. In : Rattan : Current research issues and prospects for conservation and sustainable development. Rome, Italie, Fao, Non-Wood Forest Product Series 14, 272 p.

TAYLOR L. 2005. Açaí (Euterpe oleracea). Tropical Plant Database. Raintree Nutrition. http://www.rain-tree.com/acai.htm TESORO F. O., 2002. Rattan resources of the Philippines, their extent, production, utilization and issues on resource development. In : Rattan : Current research issues and prospects for conservation and sustainable development. Rome, Italie, Fao, Non-Wood Forest Product Series 14, 272 p. VANTOMME P., WALTER S., 2003. Opportunities and challenges for non-wood forest products certification. In : La forêt, source de vie : Actes du XIIe Congrès forestier mondial, 21 28 septembre 2003, Québec, Canada.

VANTOMME P., 2004. Extraction of nonwood forest products. In : Vinceti B., Amaral W., Meilleur B. (éd. ). Challenges in managing forest genetic resources for livelihoods : examples from Argentina and Brazil. Rome, Italie, International Plant Genetic Resources Institute.

VANTOMME P, GÖHLER D., N'DECKERE-ZIANGBA F., 2004. Contribution of forest insects to food security and forest conservation : The example of caterpillars in Central Africa. ODI Wildlife Policy Briefing, 3. www.odi.org.Royaume-Uni/resources/download/2426.pdf

WORLD AGROFORESTRY CENTRE, AGROFORESTREE DATABASE, 2010. Acacia senegal. http://www.worldagroforestrycentre.org/ SEA/Products/AFDbases/AF/asp/SpeciesInfo.asp?SpID=108 